\title{
Plant Electrical signals based on wavelet transform and self similarity feature detection
}

\author{
Changcheng $\mathrm{Li}^{1, \mathrm{a}}$, Laiwu Yin ${ }^{2, \mathrm{~b},{ }^{*}}$ and Shuyun $\mathrm{Cai}^{3, \mathrm{c}}$ \\ ${ }^{1}$ College of Electrical and Information Engineering, Ji Lin Agricultural Science and Technology \\ College, Jilin, 132101, China \\ ${ }^{2}$ Department of Scientific Research JiLin Agricultural Science and Technology College, Jilin, \\ 132101, China \\ ${ }^{3}$ College of mathematics and statistics, Beihua University, Jilin, 132101, China \\ ajilinlcc@sina.com, ${ }^{b}$ yinlaiwu@163.com, ${ }^{, j i l i n l c c @ s i n a . c o m t ; ~}{ }^{*}$ Corresponding Author
}

Keywords: Continuous wavelet transform, Plant electrical signals, self similarity, feature detection

\begin{abstract}
According to the self similarity of plant electrical signal (fractal feature), changes of plant electrical signal amplitude a moment with the physical environment and mutation, causing plant electrical signal is not continuous. Electrical signal fractal characteristics of plant changes along with the time development, but at some point, it does not change with time change. This paper adopts the wavelet coefficient and self similarity relationship, through the index of self similarity calculation between plant electrical signal and wavelet to obtain the wavelet decomposition. Self similarity index is large, and plant electrical of the self similar degree are high. The simulation experiment results show that the self similarity index diagram after wavelet decomposition display can be found in many scales, the wavelet coefficients are very similar looking, providing a new idea for the detection of plant electrical signal characteristics of the physical environment.
\end{abstract}

\section{Introduction}

Generating plant electrical signal is the electrical of plant cell or tissue in greater volatility and spread to the surrounding cells, tissues or organs results, when the volatility change electrical enough to be recorded in time. Then the formation of electrical signals can be in an important physiological signal to transmit information in the plants, containing a large number of plants physiological information can reflect the growth status of plant itself and change the physical environment. Extraction of electrical signal evoked by using the method of combining independent component analysis and wavelet de-noising algorithm[1]; high frequency use wavelet multiresolution analysis method to process the signal -- noise part, respectively, simulation signal and real signal de-noising, compared to before and after noise signal SNR performance index by Daubechies (DB5) de-noising method of wavelet analysis[2]; The signal in the Matlab spectrum analysis, and then using the wavelet toolbox of DB5 is found suitable for the analysis of optimal wavelet basis of ABR signal spectrum analysis results, combined with the proposed wavelet coefficient selection algorithm is proposed[3]; the independent component analysis (ICA) and wavelet analysis combined with the method of extraction of evoked electrical (ERPs) signal [4]; modern science and technology and the theory of traditional Chinese medicine combined with analysis method, put forward a kind of human body acupoint electric electrical signal based on wavelet neural network technology [5].

Fractal characteristics of the wavelet transform is employed to decompose the plant research electrical signal, the wavelet coefficient is larger, Then the gray level is deeper, generation after wavelet decomposition approximation plant electrical signals can fractal characteristics.

\section{The continuous wavelet transform}

Telescopic translation based $\Psi \mathrm{a}, \mathrm{x}(\mathrm{t})$ continuous transformation is a continuous wavelet function in the time domain and frequency domain. It is the window of changes with frequency, so as to 
realize the low frequency components of using large window, and analysis conforms to the laws of nature by hour window of high frequency components. The wavelet transform method processing plant electrical signals will be widely application.

\section{Continuous wavelet function}

Wavelet (wavelet transform), a small region of the wave, is a special kind of finite length and an average of 0 waveforms. $\Psi(\mathrm{t})$ A square integrable function, that are $\Psi(\mathrm{t})=\mathrm{L} 2(\mathrm{R})$, if the Fourier transform $\Psi(\omega)$ to meet the conditions [6]:

$$
C_{\psi} \int_{R} \frac{|\psi \hat{(\omega)}|^{2}}{|\omega|} d \omega<\infty
$$

It is $\Psi(\mathrm{t})$ called a wavelet or wavelet generating function, now called formula (1) for the admissible condition of wavelet function.

\section{The advantages of wavelet transform}

Fourier analysis of sine wave no limitation in time, from negative infinity to positive infinity, the signal is decomposed into the superposition of a series of different frequency sine wave; but the wavelet transform tend to irregular and asymmetric. The signal is decomposed into the superposition of a series of wavelet transform function, and these wavelet functions are composed of a mother wavelet transform function through translation and scaling to. In the physical world of information, using wavelet transform function does not rule to approximate the plant electrical signals sharp change obviously better than the sine curve smooth. Therefore, characteristics of plant electrical local signal by wavelet transform function to approach than smooth sine function approximation is good. At this point the wavelet transform function alternating "volatility", namely the DC component for the obvious advantage of characteristic zero in the treatment plant electrical signals, using Meyer wavelet as an example, the three layer decomposition of plant electrical signal waveform changes is as shown in fig. 1.

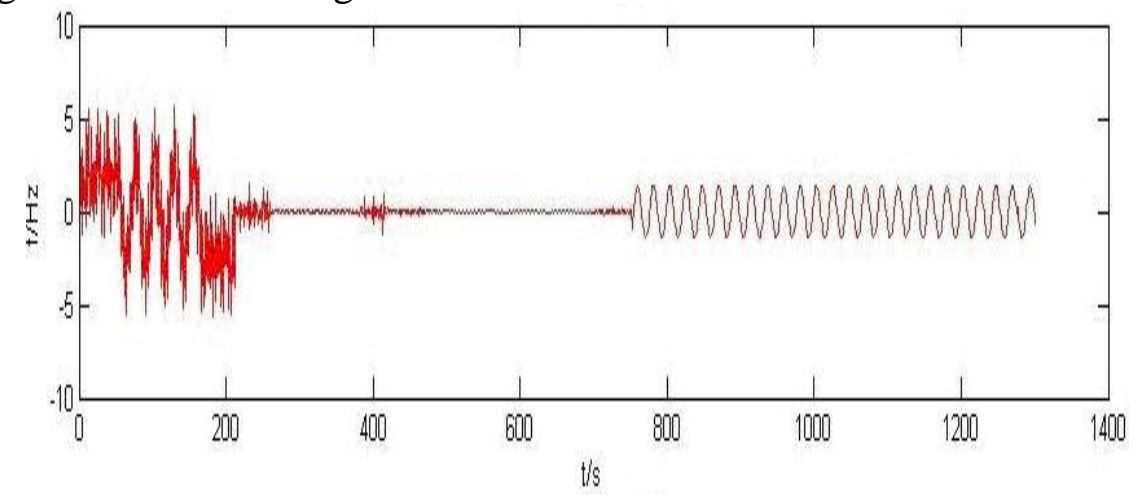

Fig. 1: Three-level decomposition of weak crops signals with DMeyer wavelet

\section{Lifting Scheme of Wavelet Transform}

The transforms of S, ST, and S+P may be seen as the special cases of Sweden's lifting scheme. The process of wavelet transform based on lifting scheme may be divided into splitting, predicting, updating and optimizing [7].

i) Splitting (Lazy wavelet or Polyphase wavelet transform)

The original signal $S \mathrm{j}, \mathrm{k}$ is divided into two disjoint subsets: $\mathrm{Sj}+1, \mathrm{k}$ and dj+1,k and the original signal $S j, k$ is generally split into even and odd series, that is $\operatorname{split}(S j, k)=(S j, 2 k, S j, 2 k+1)=(S j+1, k$, $\mathrm{dj}+1, \mathrm{k})$.

ii) Predicting (Dual Lifting Step)

As dj+1,k may be predicted with $\mathrm{Sj}+1, \mathrm{k}$ according to the relativity between data, a prediction operator, which is irrelevant to data set structure to make $\mathrm{dj}+1, \mathrm{k}=\mathrm{P}(\mathrm{Sj}+1, \mathrm{k})$ with the differential 
value between $d j+1, k$ and prediction value $P(S j+1, k)$ to replace $d j+1, k$, and the differential value represents the closeness between the above two values. If the prediction is reasonable, the differential value data set will consist of less information than the original subset $\mathrm{dj}+1, \mathrm{k}$.

iii) Updating (Update Lifting Step)

As some global properties (e.g. average value) of the coefficient subset generated from the above two steps are not consistent with those from the original data, the updating process will be applied to use operator $U$ to generate a better subset $S j+1, k$ to maintain some features of the original data set $\mathrm{Sj}, \mathrm{k}$, and $\mathrm{Sj}+1, \mathrm{k}$ may be defined as follows: $\mathrm{Sj}+1, \mathrm{k}=\mathrm{Sj}, 2 \mathrm{k}+1+\mathrm{U}(\mathrm{dj}+1, \mathrm{k})$.

After being divided through the processes of splitting, predicting and updating, $\mathrm{Sj}+1, \mathrm{k}$ can be divided into $d j+2, k$ and $S j+2, k$. The wavelet transform of the original data $S 0, k$ will be demonstrated as $\{\mathrm{SJ}, \mathrm{dJ}, \mathrm{dJ}-1, \ldots, \mathrm{d} 1\}$ after $\mathrm{J}$ times of division, and SJ, among them, represents the low frequency part of the signal, and $\{\mathrm{dJ}, \mathrm{dJ}-1, \ldots, \mathrm{d} 1\}$ represents the high frequency part of the signal.

iv) Optimizing lifting step

Dual lifting step and update lifting step may be alternatively used to improve the properties of wavelet transform according to the actual situation. The forward transform algorithm based on lifting scheme may be written as:

$$
\begin{aligned}
& S_{j+1, k}^{0}=S_{j, 2 k} \\
& d_{j+1, k}^{0}=S_{j, 2 k+1}
\end{aligned}
$$

v) Dual Lifting Step

$$
d_{j+1, k}^{(i)}=d_{j+1, k}^{(i-1)}-\sum_{m} p_{m}^{(i)} S_{j+1, k-m}^{i-1}
$$

vi) Update Lifting Step

$$
S_{j+1, k}^{(i)}=S_{j+1, k}^{(i-1)}-\sum_{m} u_{m}^{(i)} d_{j+1, k-m}^{i-1}
$$

After update lifting step and dual lifting step $M$ pair, even sample points, combined with scale factor $n_{1}, n_{\mathrm{h}}$ turn into low-pass coefficient, and odd sample points into high-pass coefficient, that is

$$
\begin{aligned}
& S_{j+1, k}=n_{l} S_{j+1, k}^{(M)} \\
& d_{j+1, k}=n_{h} d_{j+1, k}^{(M)}
\end{aligned}
$$

Inverse transform may be written as:

$$
\begin{aligned}
& S_{j+1, k}^{(M)}=\frac{S_{j+1, k}}{n_{l}} \\
& d_{j+1, k}^{(M)}=\frac{d_{j+1, k}}{n_{h}} \\
& S_{j+1, k}^{(i-1)}=S_{j+1, k}^{i}+\sum_{m} u_{m}^{(i)} d_{j+1, k-m}^{(i)} \\
& d_{j+1, k}^{(i-1)}=d_{j+1, k}^{i}+\sum_{m} p_{m}^{(i)} S_{j+1, k-m}^{(i-1)}
\end{aligned}
$$


Generally, $M$ is called the number of lifting steps, and $n_{\mathrm{l}}, n_{\mathrm{h}}$ is called normalization factor with $n_{l} \times n_{h}=1$ and the different biorthogonal wavelet leads to the different value of $n_{l}, n_{\mathrm{h}}$. The orthogonally transformed normalization process is shown in Fig. 2 (with three-level wavelet transform as the example).

\begin{tabular}{|l|l|l|l|}
\hline$\times n_{l}^{6}$ & $\times n_{l}^{5} \times n_{h}$ & \multirow{2}{*}{$\times n_{l}^{3} \times n_{h}$} & \multirow{2}{*}{$\times n_{l} \times n_{h}$} \\
\cline { 1 - 2 }$\times n_{l}^{5} \times n_{h}$ & $\times n_{h}^{4} \times n_{h}^{2}$ & & \\
\cline { 1 - 2 }$\times n_{l}^{3} \times n_{h}$ & $\times n_{l}^{2} \times n_{h}^{2}$ & \\
\cline { 1 - 2 }$\times n_{l} \times n_{h}$ & & $\times n_{h}^{2}$ \\
\hline
\end{tabular}

Fig. 2: Realization process of normalization factor of lifting scheme

The final even and odd sample points:

$$
\begin{aligned}
& S_{j, 2 k}=S_{j+1, k}^{0} \\
& S_{j, 2 k+1}=d_{j+1, k}^{0}
\end{aligned}
$$

The lifting scheme is usually applied to realize integer to integer wavelet transform in practical application.

$$
\sum_{m} p_{m}^{(i)} S_{j+1, k-m}^{(i-1)}, \sum_{m} u_{m}^{(i)} d_{j+1, k-m}^{(i)}
$$

May be written as

$$
\left\lfloor\sum_{m} p_{m}^{(i)} S_{j+1, k-m}^{(i-1)}\right\rfloor,\left\lfloor\sum_{m} u_{m}^{(i)} d_{j+1, k-m}^{(i)}\right\rfloor
$$

to carry out the integer operation.

\section{Self similarity of plant electrical signal}

In the early 1980s, Leland et al first explicitly put forward that the network data flow exists in the self similar phenomenon, which were measured and analyzed in view of some existing network in the world. It found that regardless of how the network topology and traffic, network data flow can be detected by the self similar characteristic. At this time, assuming that the physical world plant electrical signals of self similarity, it represents a part of itself and it was completely or almost similar. If saying a plant electrical signal curve is self similar, i.e., every part of the curve is a small and similar to it. Plant electrical signals for burst not definite length, showed the same burst characteristics in different time scales, signal transduction in plant electrical between the signals is long range dependent (long dependence), can be smoothed away. For a stationary random process of $\mathrm{X}=(\mathrm{Xt}: \mathrm{t}=0,1,2,3 \cdots)$. The autocorrelation function satisfying the RK $\sim \mathrm{K}-1 \mathrm{~L} 1(\mathrm{~K})$, when $\mathrm{k}$ approaches infinity, the $0<$ beta $<1$, L1 is a slowly varying function, i.e. on all $\mathrm{X}>0$; were stacked on the $X$, time sequence stacking to produce for $X(m)=(X k(m): k=1,2,3 \cdots)[8]$.

This paper describes the self similarity of plant electrical signals in the physical world, from the structure or process of spatial scales or different time scale detection of plant electrical signal characteristics. Plant electrical signals of self similarity has a more complicated form of expression, rather than local amplification of certain multiple to simply and the overall total coincidence.

\section{Test Results}

By means of the continuous wavelet transform algorithm of signal self similar feature detection, with a column of plant electrical signal as the detection object, generating self similar waveform as shown in Fig. 3 the continuous wavelet coefficients results are as shown in Fig. 4. 


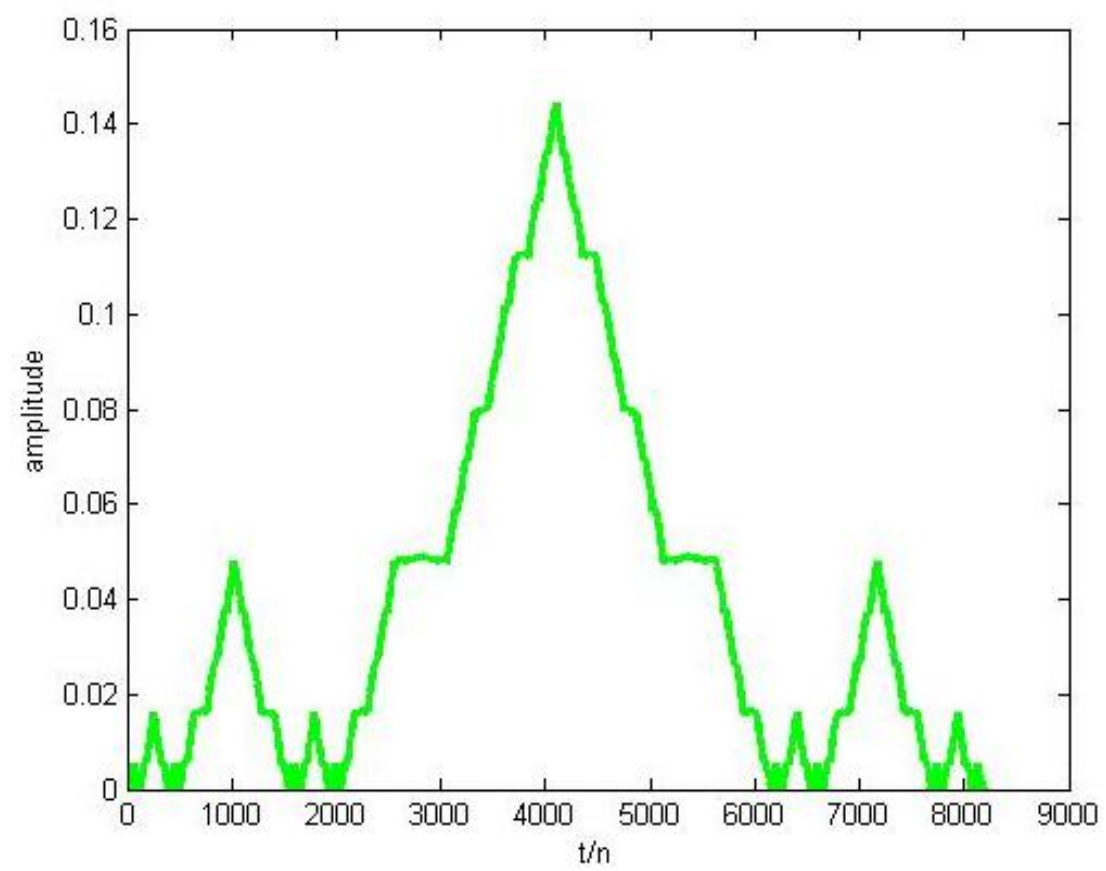

Fig.3: plant electrical signals of self similar waveform

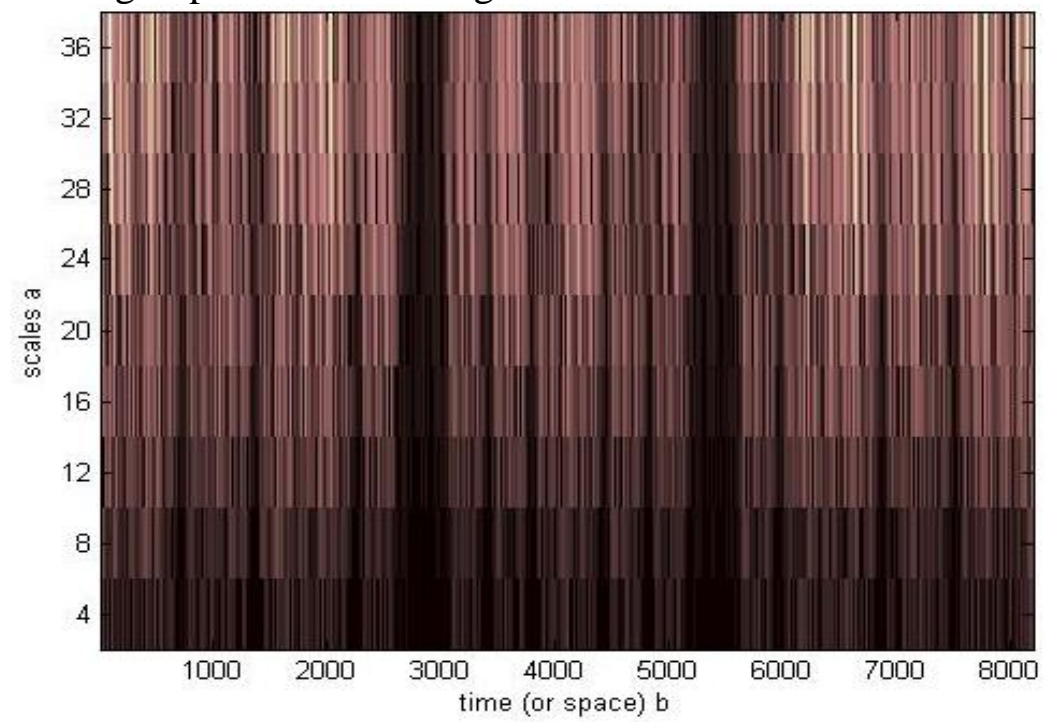

Fig. 4: after continuous wavelet transforms plant electrical signals

From Fig. 4, after the self similarity index diagram of continuous wavelet transform decomposition shows, in many scales will find that wavelet transform coefficients seem very similar. In addition, the display on vertical axis line is due to the self similarity of plant electrical signal generated, wavelet coefficient is big, gray plant electrical signals appear more deep.

\section{Conclusion}

Plant electrical signals with uncertain nonlinear and non-stationary signal, its frequency and weak, fragmented wave is associated with high frequency and low amplitude, so the high frequency components strength will be greater. According to the electrical signal in plant models under different scales of continuous wavelet transform of the self similar feature detection, plant electrical signals will be extracted to get the real signal by averaging method, based on the comparison of time and scale before and after the two kinds of signals on, more can verify the validity of the method, which has certain practical significance study on the characteristics of plants for biological world electrical signal from the microscopic angle detection. 


\section{Acknowledgement}

This research is funded by general science and technology project of Jilin Province Department of Education. (Project No: Ji Jiao Ke characters [2014] No. 382 ${ }^{\text {nd }}$, Ji Jiao Ke characters [2011] No. $152^{\text {nd }}$ )

\section{References}

[1] L. Zou, X. G. Wang, Z. H. Ma and C. C. Yang. Simulation experiment of extracting evoked potential based on the combination of independent component analysis and wavelet analysis [J]. Journal of Clinical Rehabilitative Tissue Engineering Research, 2009 13(43) 8503-8505.

[2] L. Zou, C. L. Tao and Z. H. Wang. On De-noising of Evoked Potential Signal Based on Wavelet Transform [J]. Computer Applications and Software, 2010 27(1) 85-87.

[3] Y. N. Li and Y. Sun. Research on wavelet de-noising method of auditory brainstem response [J]. Chinese Journal of Scientific Instrument, 2010 31(3) 541-545.

[4] L. Zou, S. Y. Chen, Y. Q. Sun and Z. H. Ma. Extraction of Evoked Related Potentials by using the Combination of Independent Component Analysis and Wavelet Analysis [J]. Journal of Biomedical Engineering, 2010 27(4) 741-745.

[5] J. Li, S. Su, Y. H. Bao, W. M. Tu and H. Li. Application of Wavelet Neural Network in Human Potential Signal Analysis [J]. Control Engineering of China, 2013 20(1) 106-114.

[6] C. C. Li, C. J. Xie and S. Li et al: Lossless Hyper-Spectral Image Compression Based on XCJRCT, Discrete Wavelet Transform and Set Partitioning In Hierarchical Trees Coding, 2011 International Conference on Mechatronic Science, Electric Engineering and Computer[C]. 2011 11(A) 1010-1014.

[7] Xie, C. J., Lifting Scheme, in Wavelet analysis theory and engineering application, Changchun, China: Northeast Normal Univ. Press, Changchun, 2007.

[8] C. C. Li, L. W. Yin, D. Chen and X. H. Tang. Threshold of Denoising Weak Electrical Signals in Plants from Daubechies Wavelet Transform, 2013 International Conference on Computer Sciences and Applications[C], 2013 8(13) 600-603. 